\title{
BMC Emergency Medicine reviewer acknowledgement 2015
}

Giulia Mangiameli

\section{Contributing reviewers}

The editors of BMC Emergency Medicine would like to thank all our reviewers who have contributed to the journal in Volume 15 (2015)

\author{
Imo Aisiku \\ USA \\ Samina Ali \\ Canada \\ Jose Alonso-Iñigo \\ Spain
}

Dug Andrusiek

Canada

Ali Ardalan

Iran

Bonnie Arquilla

USA

Marc Auerbach

USA

Julie Bembenisty

Israel

Anthony Bleetman

UK

Andrew Boyd

USA

Janet Bray

Australia

Alexandra Brazinova

Austria

Mette Brekke

Norway

\begin{abstract}
Jane Brice
USA
\end{abstract}

Georges Brousse

France

Lane Burgette

USA

\section{Pietro Caironi}

Italy

Phillipo Leo Chalya

Tanzania

Yves Chaput

Canada

Mario Chiavarelli

Italy

Stanford Chihuri

USA

Jeff Clawson

USA

Shelley Cox

Australia

Christopher Coyne

USA

Janet Curtis

Australia

Jihong Dai

China

\author{
Andrew Dawson \\ Australia
}

Conor Deasy

Ireland

\section{Antonio Dell'Anna}

Italy

Scott Devenish

Australia

Esperanza Diaz

Norway

Bridget Dicker

New Zealand

Pratik Doshi

USA

Bin Du

China

Jhodie Duncan

Australia

Kylie Dyson

Australia

Paul Eustace

Ireland

Stephanie Fincham-Campbell

UK

Gerry Fitzgerald

Australia

Correspondence: giulia.mangiameli@biomedcentral.com

BioMed Central, Floor 6, 236 Gray's Inn Road, LondonWC1X 8HBUnited

Kingdom 
Roberto Forero

Australia

Federico Franchi

Italy

Erika Frischknecht Christensen

Denmark

Cornelia Genbrugge

Belgium

Georgios Giannakopoulos

Netherlands

Scott Goldberg
USA

Morris Gordon

UK

Arthur Greenberg

USA

Patrick Henn

Ireland

Falco Hietbrink

Netherlands

Nico Hoogerwerf

Netherlands

Amir Jalali

Iran

Anna Jönsson

Sweden

John Kalbfleisch

USA

Julie Kanter-Washko

USA

Kianoush Kashani

USA

David Katz

USA

Bory Kea

USA

Jakub Kenig

Poland

Sankalp Khanna

Australia

Kosaku Kinoshita

Japan

Frederick Kofi Korley

USA
Emmanuel Lagarde

France

Melissa Langhan

USA

Outi Lapatto-Reiniluoto

Finland

Andrew Chee Keng Lee

UK

Resa Lewiss

USA

Li Luo

China

Mary Mackesy-Amiti

USA

Ibrahim Mahmoud

Australia

Marek Majdan

Slovakia

Sheryl Martin-Schild

USA

Rahim Moineddin

Canada

Dan Mullany

Australia

Sebbane Mustapha

France

Hüseyin Narcı

Turkey

Sagar Nigwekar

USA

Alexander Olaussen

Australia

Don Olson

USA

Kent Olson

USA

Peter O'Meara

Australia

Sean P. Kelly

USA

Anna Paldy

Hungary

Sukhmeet Panesar

UK
Robert Patton

UK

Roshini Peiris-John

New Zealand

Timothy Platts-Mills

USA

Senija Rasic

Bosnia and Herzegovina

Joseph Restuccia

USA

Joao Rezende-Neto

Canada

Zaccaria Ricci

Italy

Schalk Richard

Germany

Giuseppe Ristagno

Italy

Stefano Romagnoli Italy

Martin Rusnak

Slovakia

Fabio Salvi

Italy

Claudio Sandroni

Italy

Dena Schanzer

Canada

Patrick Schober

Netherlands

Michael Schull

Canada

Raghu Seethala

USA

Greene Shepherd

USA

Lorraine Sheppard

Australia

Jordan Simanjuntak

USA

Cor Slagt

Netherlands

Steve Socransky

Canada 
Eldar Soreide

Norway

Tracy Stecker

USA

Amy Stuck

USA

Kostas Stylianou

Greece

Andrew Tabner

UK

Fabio Silvio Taccone

Belgium

Benjamin Taylor

USA

Lateef Thanni

Nigeria
Lizbet Todorova

Sweden

Mahmoud Torabi

Canada

Vicken Totten

USA

Cynthia Van Der Starre

Netherlands

Kathleen Ventre

USA

Giovanni Volpicelli

Italy

Carl Von Baeyer

Canada

David Wampler

USA
Hao Wang

China

Richard Weinert

USA

Scott Weinstein

Australia

Ben White

USA

Michael Wilson

USA

Keramettin Yanik

Turkey 